\title{
Advances in Water Content Measurement With Distributed Fiber-optic Sensor
}

\author{
Sibel Pamukcu ${ }^{1}$, Sylvain Texier $^{2}$ and Jean Toulouse ${ }^{3}$ \\ ${ }^{1}$ Lehigh University, Department of Civil and Environmental Eng., 13 East Packer Ave., Fritz Engineering \\ Laboratory, Bethlehem, Pa 18015, PH (610) 758 3220; FAX (610) 758 6405; email: sp01@lehigh.edu \\ ${ }^{2}$ Lehigh University, Department of Civil and Environmental Eng., 13 East Packer Ave., Fritz Engineering \\ Laboratory, Bethlehem, Pa 18015, email: syt3@1ehigh.edu \\ ${ }^{3}$ Lehigh University, Department of Physics, 16 Dewing Lewis Laboratory, Bethlehem PA 18015, PH (610) 758 \\ 3960; e-mail: jt02@lehigh.edu
}

\begin{abstract}
The design and functionality of an optical sensor for distributed measurement of water content in soil is presented. Sensing is based on the characteristics of Brillouin scattering in optical fiber coupled with the characteristics of a hydrophilic polymer used as transducer. The distributed sensing is possible at discrete positions along a single fiber embedded in low permeability media. Experiments with a prototype sensor demonstrated measurement of water-content up to $30 \%$ in compacted clay. Swelling and drying of the polymers showed that the water-content measurements are fully reversible.
\end{abstract}

\section{Introduction}

On-line monitoring of civil-infrastructure, subject to vibrations or environmental effects, is of great interest to civil engineers. The distributed sensing capability using Brillouin scattering optical fibers offers a viable method to continuously monitor buried structures, as well as those on surface. The Brillouin scattering in standard optical fibers makes it possible to obtain strain measurements by time domain reflectometry at intermitted positions along a single fiber subject to thermal or mechanical loading. The entire fiber length - in meters or kilometers - can be used for sensing with spatial resolution of measurement varying from about several centimeters to several meters (Brown et al. 1999).

Strain and temperature sensing capability of Brillouin Optical Time Domain Reflectometry (BOTDR) has been demonstrated successfully for long gauge lengths for structural monitoring (Bao et al. 2001, Kim et al. 2002, Mitra 1999; Thevenas, et al. 1999; Inaudi 2005). The sensor requires access to only one end of the fiber, which simplify the fiber layout on the structure and uses a single laser source preventing measurement errors from two laser emission fluctuations. The measurement technique enables the use of low birefringence optical fibers, which are economical compared to polarization maintaining fibers. Low birefringence fibers can be spliced with inconsequent power losses such that fiber sections can be affixed on separate structural elements and spliced together as the complete structure is assembled.

The Brillouin based strain sensing capability of the fiber can also be used to detect other variations in the host material, such as temperature, liquid and chemical content (Moran et al., 2000; Texier and Pamukcu, 2003). In these, the fiber is hitched or bonded to a selective polymer material in direct contact with the surrounding medium. The polymer reacts to the surrounding (i.e., moisture, $\mathrm{pH}$, chemical) by volume expansion as it selectively absorbs the target compound. Expansion of the polymer produces tangential, axial or radial stresses on the fiber, hence axial straining and Brillouin signal. A single line of fiber stringed with polymer mass detectors at predetermined spacing can be embedded into a host medium to detect target chemical(s) by linear positioning of the fiber. Other fiber optic sensors developed as a liquid and moisture detectors include Fibre Bragg Grating (FBG) based probes providing single point values with a limited measurement range of up to $4 \%$ (Laylor et al. 2002; Kunzler et al. 2003). Some of the distributed fiber optic sensors based on Optical Time Domain Reflectometry (OTDR) provide accurate information on liquid detection but they can be limited on quantitative measurements (Moran et al. 2000; Yuan et al. 2001; Buerck et al. 2001).

The sensor described in this paper is an assembly of optical fiber and a hydrophilic polymer used as a mass transducer, for continuous detection and quantification of moisture over extended subsurface environments. A thin polymer ring is used to stretch the fiber at the sensing location as a function of polymer's volume expansion. Typical subsurface applications may include monitoring of moisture underneath paved surfaces, inside road embankments, wall backfills, waste containment facilities, and at soil-structure interfaces. 


\section{Background}

\section{Brillouin scattering based sensing}

In a dielectric material like the silica of an optical fiber, material tends to compress in the regions of high intensity electrical field (electrostriction). Periodic compression zones create a density wave moving in the material (Malinder and Proctor 1964). If the speed of this wave corresponds to the speed of sound in the material, an acoustic wave is created. Brillouin Scattering results from the scattering of the incident (pump) light by the acoustic waves. The scattered light is shifted downward in frequency to the Stokes frequency. The separation between the frequencies of the incident and scattered light is called the Brillouin frequency shift, $v_{B}$, given as follows:

$$
v_{B}=\frac{\omega_{p}-\omega_{S}}{2 \pi}=\frac{2 n V_{A}}{\lambda_{p}}
$$

where, $\omega_{\mathrm{p}}$, and $\omega_{\mathrm{S}}$, are the frequencies of the pump and the Stokes waves, $V_{A}$ and $n$ are the acoustic velocity and the refractive index of the fiber core, respectively; and $\lambda \mathrm{p}$, the wavelength of the pump lightwave. According to equation (1), the Brillouin frequency shift, $v_{\mathrm{B}}$ is directly proportional to the acoustic velocity of the optical fiber. The elastic properties of silica make any induced strain result in locally modified material density, hence change in its acoustic velocity, $V_{A}$. When the refractive index, $n$, of the fiber is known, by measuring the Brillouin shift $v_{B}$, one can determine the local change in the acoustic velocity, hence the induced strain. Knowing the proportionality coefficient between the two quantities, one can obtain the isothermal strain corresponding to $v_{\mathrm{B}}$ measured at discrete points along the fiber, as given in equation (2):

$$
\varepsilon-\varepsilon_{0}=\frac{v_{B}-v_{B\left(\varepsilon_{0}\right)}}{C v_{B\left(\varepsilon_{0}\right)}}
$$

where, $\mathrm{C}$ is the dimensionless proportionality coefficient that describes the collective change in refractive index, elastic modulus, mass density and Poisson's ratio of the silica core subjected to strain; and $v_{\mathrm{B}(\varepsilon 0)}$ is the Brillouin frequency of the fiber at a reference strain, $\varepsilon_{0}$.

\section{Methods of Investigation}

\section{Calibration of fiber strain with Brillouin frequency shift}

For calibration of the test fiber (Corning SMF28) as a distributed strain sensor, $86.4 \mathrm{~cm}$ section of $120 \mathrm{~m}$-fiber was secured at two end points. The fiber was strained incrementally (increments of $230 \mu \varepsilon$ ) using a translation stage with measurement accuracy of $10 \mu \varepsilon$. Next, the same fiber was set in a 3-pulley system and loaded with several calibrated weights- from $1 \mathrm{~kg}$ to $7 \mathrm{~kg}$ in $1 \mathrm{~kg}$ increments. The stress in the fiber was computed using the applied load and the cross-sectional area of the fiber (core and cladding diameter $125 \mu \mathrm{m}$ ). The corresponding Brillouin frequency shift was recorded for each applied stress. The variation of the independently measured Brillouin frequency shift with the applied strain and the stress are shown in Figure 1, with a computed proportionality constant, $\mathrm{C}$ of 4.3. Since each frequency corresponds to a unique stress and strain in the fiber, a one-to-one relation is found between the independently measured values. Hence, the Brillouin determined stress-strain relation of the test fiber resulted in elastic constant of $79.7 \mathrm{GPa}$, which is slightly higher than that of pure silica fiber (72GPa). This deviation is expected since most of the commercial optical fibers are doped with property enhancing elements (i.e., germanium).

\section{Prototype distributed fiber optic water content sensor}

The prototype design of the water content sensor consisted of using pure hydrophilic polymers hinged to the Brillouin detecting optical fiber. The polymer reacts to the surrounding moisture by swelling as it selectively absorbs the water. The physical coupling of the polymer and the optical fiber produce local axial strains in the fiber, hence Brillouin signals. A single line of fiber stringed with these hydrophilic mass detectors at predetermined spacing sense changes in local water content by linear positioning of the fiber. The coupled assembly of polymer ring and the optical fiber essentially work to convert one form of energy to another, hence 
it works as a transducer. Taking advantage of this analogy, the sensor assembly discussed here will be referred to as "transducer" in this text.

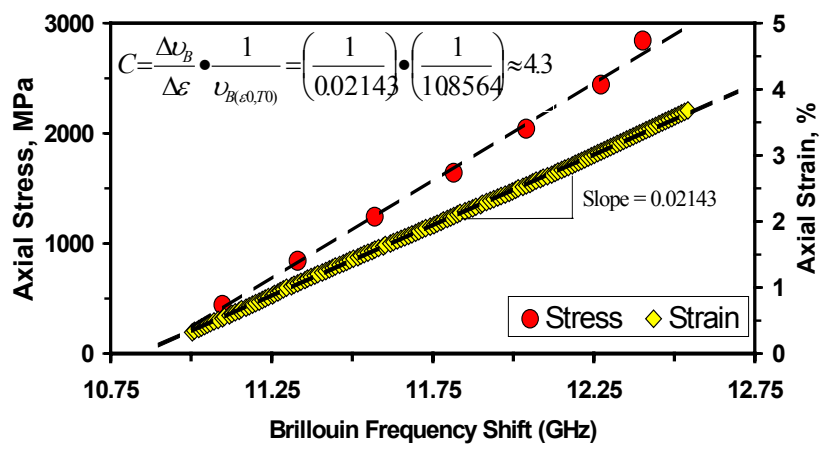

Figure 1. Brillouin frequency correlation with independent stress \& strain (Corning SMF28 fiber)

The hydrophilic polymer $\left(\mathrm{AEP} 60^{1}\right)$ used in construction of the transducers could be extruded into a rod form of any diameter by its manufacturer, as shown in Figure 2. The original hollow rods were further cut to trial size of $6.5 \mathrm{~cm} \mathrm{OD}, 5.5 \mathrm{~cm}$ ID and $0.8 \mathrm{~cm}$ width rings. The outer edge of each ring was threaded to host tightly wound $82 \mathrm{~cm}$-sections of a single stretch of fiber. The fiber was glued to the polymer at two points - at the beginning and at the exit of the loop - with high strength marine glue. A tightly fitting acrylic disc $(5.5 \mathrm{~cm}$ OD) was inserted inside the ring to prevent inward radial swelling. The volumetric expansion was observed to take place principally in the radial direction due to the orientation of the polymer chains in the material.

The AEP60 polymer was specifically manufactured for the water content detection in this experimental program. AEP materials typically expand from $38 \%$ to $400 \%$ in volume over dry volume in water. They are non-toxic and are manufactured in medical grades, approved for use in human wound care applications. This group of polymers does not swell in hydrocarbons and chlorinated solvents and has high thermal resistance. They are cross-linked to give them mechanical stability and accurate expansion characteristics. The water absorption and expansion factor can be accurately pre-defined at the formulation stage. Full expansion is reproducible over many wetting and drying cycles and is consistent over a wide range of $\mathrm{pH}$ and dissolved solid concentrations. The physical properties of the AEP60 used in this work are provided in Table 1.

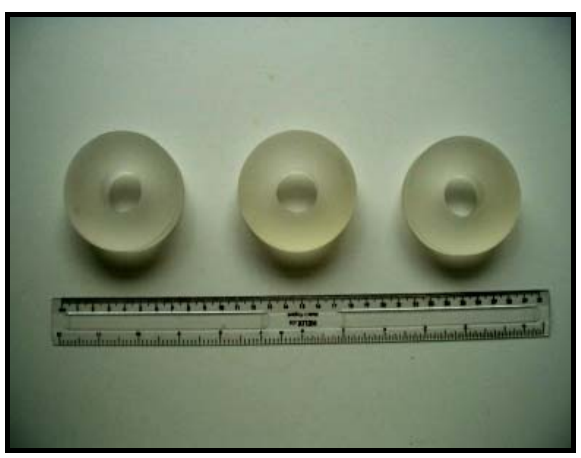

Figure 2. A photograph of the AEP60 polymer rings used in the development of the water content sensor

\footnotetext{
${ }^{1}$ IH Polymeric Products, Andrews Wright Limited, Absorption and Expansion Polymers, Kent, UK
} 
Table 1. List of typical properties for AEP polymers ${ }^{1}$

\begin{tabular}{l|c}
\hline \multicolumn{1}{c|}{ Property } & Value or Limit \\
\hline Volume Expansion, \% @ 20 C & 557 \\
- Water & 569 \\
- pH 1.0 (dil. HCl) & 579 \\
- pH 14.0 (dil. NaOH) & 569 \\
- Saline (1\% NaCl) & 1.77 \\
Linear expansion ratio, @ 20 ${ }^{\circ} \mathrm{C}$ water & 80 \\
Moisture content, \% @ 20 ${ }^{\circ} \mathrm{C}$ & $50: 50$ \\
Ratio of bound to free water & \\
Effective pore diameter, microns & 0.46 \\
- Hydrated & 0.01 \\
- Dry & 21 \\
Tensile modulus, MPa & 0.35 \\
- Dry & 1.16 \\
- Hydrated @ $40 \%$ strain & 310 \\
Tensile strength hydrated, MPa & 1.2 \\
Elongation to break, \% & 180 \\
Mass Density (g/cm ${ }^{3}$ ) & chars @ $220{ }^{\circ} \mathrm{C}$ \\
Temperature stability, ${ }^{\circ} \mathrm{C}$ & insoluble \\
Flammability - dry & inert \\
Particle size, microns & \\
Solubility in water & \\
Environment &
\end{tabular}

The three important aspects of the transducer configuration was to ascertain a design that would allow light to be guided through the fiber without exiting the core by refraction; to accommodate the entire gauge length of the fiber on the transducer and to provide rapid kinetics for detection and quantification. A very simple set up was used to determine the minimum bending curvature for the fiber to minimize the loss of light through bending and refraction, as shown in Figure 3. By fixing the power of the input light in the set-up, the variation of the output power could be measured with respect to the bending diameter as the diameter of the central ring was varied. Using a factor of safety of 2.8 , the working bending diameter and hence the outer diameter of the polymer ring was selected as $6.5 \mathrm{~cm}$.

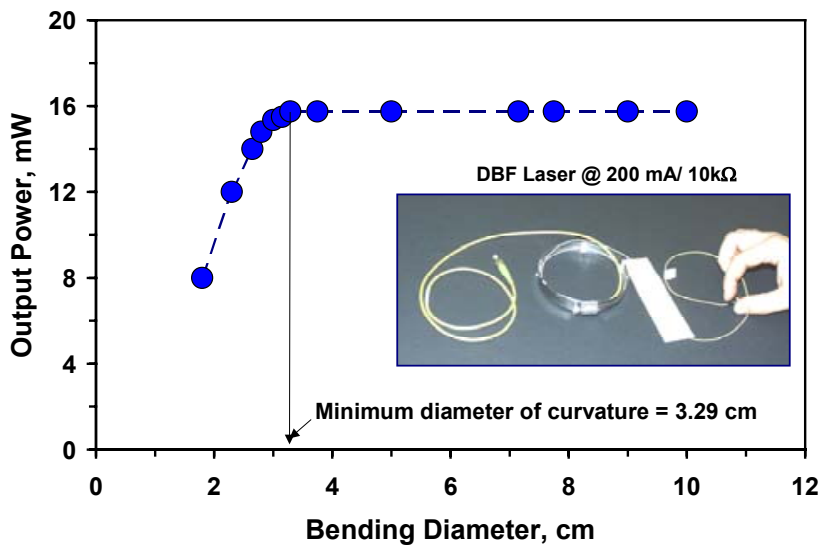

Figure 3. Assembly and test results for minimum fiber bending radius assessment

\footnotetext{
${ }^{1}$ IH Polymeric Products, Andrews Wright Limited, Absorption and Expansion Polymers, Kent, UK
} 
The width of the polymer ring was set to house the minimum gauge length $(80 \mathrm{~cm})$ of the sensing fiber when tightly wound into the threads of the ring's outer surface. The selected width of $0.8-\mathrm{cm}$ was adequate to hold four revolutions of the fiber over $83-\mathrm{cm}$ stretch. Four polymer transducers were wired along the same fiber with 10-m spools of fiber in between each transducer (see inset sketch in Figure 4). The original polymer rods were extruded with specific orientation of the polymer chains so that the principal volume expansion of the ring would be radial. In order to limit all potential expansion to outward direction, a solid acrylic disc of identical diameter $(5.5 \mathrm{~cm})$ and width of the ring hole was inserted to prevent the inward expansion. This design was employed to improve the time rate of detection and quantification of the surrounding liquid. The radial thickness of the ring $(1 \mathrm{~cm})$ was selected as a trial dimension value. Increased ring thickness was expected to provide larger volume expansion hence higher accuracy in detecting low water contents. Alternatively, thinner rings would require shorter duration to reach maximum volume expansion. Therefore thickness of the transducer could be optimised for desired outcomes, such as adjusting the lower and upper limits of detection and/or rate of quantification. In this study, the trial thickness of $1 \mathrm{~cm}$ for the AEP60 polymer rings was adequate to detect moisture in wet clay with water content as low as $5 \%$.

\section{Calibration of water content with Brillouin frequency shift}

The optical fibre at the inlet and outlet of the string of four transducers were spliced to spools of fibre on each end, and connected to a photonic and data acquisition set-up (Texier and Pamukcu, 2003). Each water transducer was embedded in a wet clay sample of predetermined water content $(5,10,20$ and $30 \%)$. The clay samples were packed in equal volume, watertight, cylindrical cells of $14-\mathrm{cm}$ diameter and $28-\mathrm{cm}$ height. The transducers were embedded at the centre of each soil cell with entry and exit holes on the chamber cap for the optical fiber. The experiments were conducted in temperature-controlled environment, at $25^{\circ} \mathrm{C}$ so that Brillouin scattering measurements were not influenced by thermal expansion or contraction of the fiber. The Brillouin shift was measured with 5 to 20 minute intervals up to the maximum observable swelling - when no significant change in Brillouin shift was recorded for three consecutive measurements. Then the transducers were removed from the soil chambers and left for open air-drying. Brillouin shift measurements were recorded with 5 to 20 minute intervals until the drying phase was completed.

The degree and rate of the volume change of the four polymer rings were mapped to local axial strains, hence to Brillouin signals over the coupled sections of the optical fiber. Since Brillouin readings could be recorded for strains as low as $10 \mu \varepsilon$, very small volume changes due to water exposure could be detected in a few minutes. Figure 4 shows the time rate of swelling and shrinkage of the hydrophilic transducers as correlated to the Brillouin signal measurements. The transducers reside on the same line of fiber, yet each is embedded in a compacted clay specimen of different water content (5\% to 30\%) as shown in the sketch (inset Fig. 4).

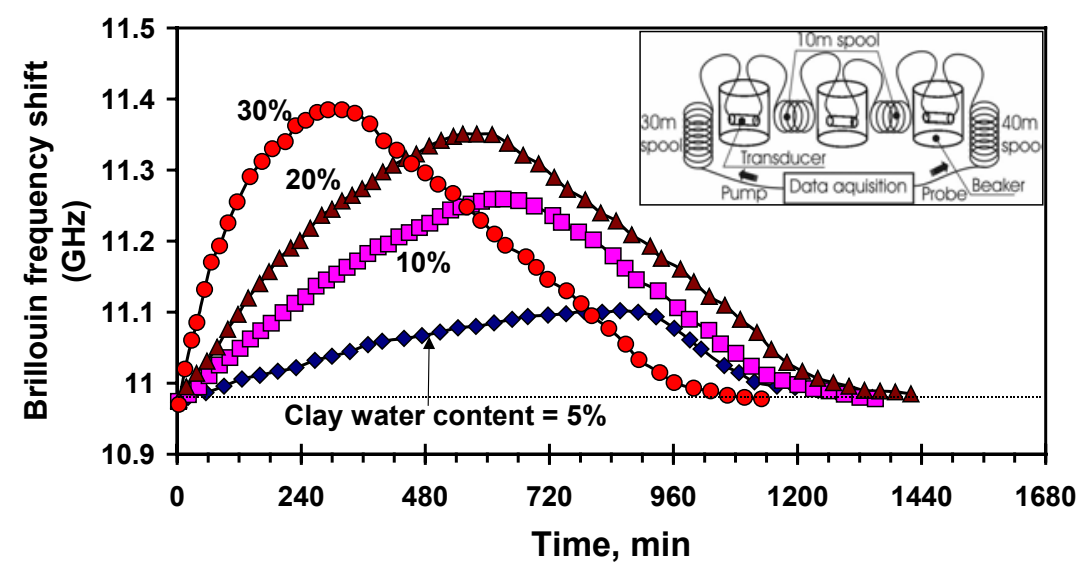

Figure 4. Brillouin signal shift as a function of swelling/shrinkage time and clay water content (Inset - sketch of experimental set up)

As observed, the maximum Brillouin signal and its rate increased with increasing soil water-content, while the duration to maximum swelling decreased. The rate behaviour was expected, as larger portion of the polymer 
surface would be in contact with water at higher soil water contents, thereby promoting faster volume expansion. It is not conclusive from the data if the peak Brillouin signal is true indicator of full swelling since the AEP type polymers can swell to multiple times of their original volume. It is highly plausible that upon completion of the initial swelling, the polymer would continue to swell at a lower rate with soil water diffusing towards the polymer. Hence it is necessary to improve the geometrical design of the transducer, possibly using separation layers, to avoid low rate swelling following the initial surge. This is important for two reasons: 1 . for accurate calibration of soil water quantification, 2. to assure that the elastic range of the fiber is not exceeded to avoid sudden breakage by over stretching. The full reversal of the volume expansion, hence contraction of the polymer ring could be detected with the reversal of the Brillouin signals, as shown in Figure 4 in the drying phase of the polymer, following the peak Brillouin signal. The rate of polymer contraction was essentially same for all transducers and the volume reduction was completely reversible showing good durability and re-usability of the sensor assembly.

\section{Conclusions}

The prototype fiberoptic water sensor meets the requirements of distributed monitoring of subsurface moisture by measuring the water intake of water-sensitive polymers coupled to the optical fibre at discrete locations along the entire length of a single fibre embedded in host medium. The results shown in this experimental work demonstrate the capability of BOTDR to measure distributed water contents in low permeability soils. The transducer design and its properties can be modified to improve the kinetics of volume expansion and the measurable water content range. In this study, measurements of water-contents up to $30 \%$ were possible. Reversibility of sensing was also demonstrated with swelling and drying phases of the polymer and the corresponding Brillouin shift response.

\section{References}

Bao, X., DeMerchant, M., Brown A., and Bremner, T. (2001). "Tensile and compressive strain measurement in the lab and field with the distributed Brillouin scattering sensor." Journal of Lightwave Technology, 19:1698.

Buerck, J., Roth, S., Kramer, K., and Mathieu, H. (2001). "OTDR distributed sensing of liquid hydrocarbons using polymer-clad optical fibers'." Proc. of The Second Int. Symp. and Workshop on Time Domain Reflectometry for Innovative Geotechnical Applications, C. H. Dowding, ed., Academic, Evanston, Il., 496-509.

Inaudi, D., (2005). "Development of Distributed Strain and Temperature Sensing Cables." $17^{\text {th }}$ Int. Conf. On Optical Fiber Sensors, Bruges, Belgium, May 23-27, 2005, Paper No.Th3-3.

Kim, S.H., Lee, J., and Kwon, I. (2002). "Structural monitoring of a bending beam using Brillouin distributed optical fiber sensors." Smart Materials and Structures, 11:396.

Kunzler, W., Calvert, S.G., and Laylor, M., (2003). "Measuring Humidity and Moisture with Fiber Optic Sensors," Proceedings of SPIE, Vol. 5278, p.86.

Laylor, M., Calvert, S., Taylor, T., Schulz, W., Lumsden, R., and Udd, E. (2002). "Fiber Optic Grating Moisture and Humidity Sensors," Proceedings of SPIE, Vol. 4694, p. 210.

Mallinder, F. and Proctor, B. (1964). "Tensile strain dependence of Brillouin frequency shift in silica optical fibers," Photon. Technol. Lett., 5(4):91-103.

Mitra, A. (1999). "Emerging Needs in Japan for Health Monitoring Technologies in Civil and Building Structures." $2^{\text {nd }}$ IWSHM, Stanford University, Sept. 8-10.

Moran, M., Thursby, C., Pierce, G., Culshaw, S., and Graham, B. (2000). "Distributed fiber optic sensors for humidity and hydrocarbon detection." Proc. of SPIE, 3986: 342-351.

Texier, S. and Pamukcu, S. (2003). "Water-content measurement in sub-surface with a distributed Brillouin scattering fiber-optic sensor." Proc. of SARA 2003, $12^{\text {th }}$ Pan-American Con. on Soil Mech. and Geotechnical Eng. \& $39^{\text {th }}$ US Rock Mech. Symp., MIT, Ma, June 22-26.

Thevenaz, L., Facchini, M., Fellay, A., Robert, P., Inaudi, D., and Dardel, B. (1999). "Monitoring of large structure using distributed Brillouin fiber sensing." Proc. of SPIE, 3746:345.

Yuan, J., El-Sherif, M.A, MacDiarmid, A.G., and Jones W.E. Jr. (2001). "Fiber optic chemical sensors using a modified conducting polymer cladding," Adv. Env. And Chem. Sensing Tech, Proceedings of SPIE, Vol 4205, pp. 170-179. 